\title{
Submillimeter-Wave Phasor Beam-Pattern Measurement Based on Two-Stage Heterodyne Mixing With Unitary Harmonic Difference
}

\author{
Yuh-Jing Hwang, Member, IEEE, Ramprasad Rao, Rob Christensen, Ming-Tang Chen, and \\ Tah-Hsiung Chu, Member, IEEE
}

\begin{abstract}
A near-field phasor beam measurement system is developed for the characterization of heterodyne receiver optics at submillimeter-wave frequencies. The system synthesizes a pair of submillimeter-wave signals as the RF and local oscillator (LO) sources from common reference sources. The synthesized harmonic numbers of the RF and LO sources are arranged with difference by one, which makes this a new configuration with a unitary harmonic difference. The coherent RF and LO signal are down-converted by the receiver under test, then mixed with the microwave-frequency common reference signal to generate the second-order IF signal around $100 \mathrm{MHz}$ for amplitude and phase comparison. The amplitude and phase fluctuation of the measurement system at $683 \mathrm{GHz}$ is within $\pm 0.2 \mathrm{~dB}$ and $\pm 4^{\circ}$ in a 1-h period, respectively. The system dynamic range at 683 and $250 \mathrm{GHz}$ can be as high as 43 and $47 \mathrm{~dB}$, respectively. The system is then used to measure the receiver beam patterns at 683 and $250 \mathrm{GHz}$ with different RF transmitting probe antennas.
\end{abstract}

Index Terms-Frequency conversion, Gaussian beams, phase measurement, submillimeter-wave receivers.

\section{INTRODUCTION}

$\mathbf{W}$ ITH THE increasing demands on atmospheric observing and radio astronomical instruments, the development of the submillimeter-wave heterodyne receivers with high sensitivity and accurate pointing is required [1]-[3]. The highly sensitive heterodyne receiver can be implemented by using a nearly quantum limited superconductor-insulator-superconductor (SIS) mixer or a hot-electron bolometer mixer as the front-end [4]-[6]. For accurate pointing of the receiver optics, a useful and efficient diagnostic instrument is required to measure the beam pattern to analyze the optical parameters of the receiver at the operating frequency.

Manuscript received May 18, 2006; revised February 16, 2007. This work was supported by Academia Sinica under the Submillimeter Array of Taiwan Project and by the R.O.C. National Science Council under Grant NSC95-2752E002-004-PAE and Grant NSC95-2221-E002-086-MY3.

Y.-J. Hwang, R. Rao, and M.-T. Chen are with the Institute of Astronomy and Astrophysics, Academia Sinica Taipei 10617, Taiwan, R.O.C. (e-mail: yjhwang @asiaa.sinica.edu.tw; rrao@sma.hawaii.edu; mchen@asiaa.sinica.edu.tw).

R. Christensen is with the Submillimeter Array, Hilo, HI 96720 USA (e-mail: rchriste@sma.hawaii.edu).

T.-H. Chu is with the Graduate Institute of Communication Engineering and the Department of Electrical Engineering, National Taiwan University, Taipei 10617, Taiwan, R.O.C., and also with the Institute of Astronomy and Astrophysics, Academia Sinica Taipei 10617, Taiwan, R.O.C. (e-mail: thc@ew.ee. ntu.edu.tw).

Color versions of one or more of the figures in this paper are available online at http://ieeexplore.ieee.org.

Digital Object Identifier 10.1109/TMTT.2007.897843
A typical phasor antenna pattern measurement system consists of a coherent transmitter-receiver module, or so-called phasor network analyzer, combined with a precision scanner to compare the amplitude and phase between transmitted and received signals at different relative positions. For frequencies below $500 \mathrm{GHz}$, a commercially available millimeter-wave phasor network analyzer was introduced recently [7]. For higher frequencies below $1 \mathrm{THz}$, a millimeter-wave vector network analyzer is also available with user-reconfigurable test sets. ${ }^{1}$ Previous research on antenna or telescope-optics pattern measurement using commercially available phasor network analyzers has also been published [8]-[17], and the amplitude dynamic range can be as high as $60 \mathrm{~dB}$ at $860 \mathrm{GHz}$. However, these commercially available phasor network analyzers are based on the harmonic mixer as the down-converting device, which limits its application to the integrated measurement of the antenna system with a heterodyne receiver. To solve this problem, some research for frequency ranging from 200 to $1500 \mathrm{GHz}$ has also been published [18]-[22]. These receiver beam pattern measurement configurations typically require complicated frequency synthesis and conversion. Phase stability is an important factor in the measurement system. For a system with phase sensitive to the environmental temperature, the scanner carried RF transmitter requires an axis along the beam propagation to scan the beam pattern at different propagation positions. However, once the measurement configuration exhibits stable phase, the measured amplitude and phase data secured by scanning at a fix plan perpendicular to the beam axis are sufficient to extract the beam parameters.

In this paper, the simple, but stable measurement configuration for submillimeter-wave phasor beam pattern and receiver optics alignment described in [23] for $690 \mathrm{GHz}$ is adapted to multiple frequency measurement in Section II. The configuration only needs two frequency standards - one microwave swept frequency source and one fixed reference frequency source- to synthesize and convert the frequencies required for the measurement. When measuring the receiver systems equipped with multiple channels for different frequency bands, this configuration can be used for measuring beam pattern by replacing the transmitting antenna, rotating the polarization of the RF transmitting module, and switching the active receiver channel. A theoretical formulation of the phase stability of the measurement configuration is derived in Section III. Both the theoretical prediction and

\footnotetext{
${ }^{1}$ Type MVNA-8-350 Millimeter-Wave Network Analyzer, AB Millimetre, Paris, France.
} 
the measurement show that this method can reduce the system phase variation to as low as $\pm 4^{\circ}$ during a 1 -h period.

In Section IV, a multichannel SIS-based heterodyne receiver for the Submillimeter Array (SMA) telescope is measured as the receiver under test. Operating frequencies around 250,349, 409 , and $683 \mathrm{GHz}$ are chosen to measure the receiver front-end quasi-optics beam pattern. The measurement results show a dynamic range around $47 \mathrm{~dB}$ for the $230-\mathrm{GHz}$ band receiver and $43 \mathrm{~dB}$ for the $690-\mathrm{GHz}$ band. A formulation for extracting the optical parameters of the truncated Gaussian Beam is also derived. The extracted parameters are compatible with the theoretical prediction.

\section{Measurement ARrangement}

In order to configure the submillimeter-wave coherent transmitter-receiver module in a simpler approach with better performance, the frequency synthesis and conversion approaches of the measurement arrangements in [7]-[22] are investigated to find a method for improvement.

The millimeter-wave phasor network analyzer in [7] requires two microwave synthesizers to generate RF and local oscillator (LO) signal. An additional $20-\mathrm{MHz}$ signal source is used as phase and amplitude comparison reference. The receiver inside the test sets uses harmonic mixers as down-converters, but it is still possible to adapt it for heterodyne receiver measurement. However, even if one can apply this configuration to the receiver beam pattern measurement, the disadvantage on the coherence between RF and LO signals due to the locking frequency at only $10 \mathrm{MHz}$ limits its operation at higher submillimeter-wave frequencies. Typical operation of this kind of millimeter-wave phasor network analyzer requires 128 times averaging to reduce the random fluctuation, which leads to longer measurement time.

The other popular phasor network analyzers described in [8]-[17] provide flexible and different frequency synthesis and conversion approaches. When operating at a lower frequency, its utilizes a frequency multiplier to generate RF source and down conversion of the received RF signal to IF by harmonic mixer, which is similar to [7], for higher submillimeter-wave frequency it relies on one swept microwave synthesizer and two distinct low-frequency signal sources for phase and amplitude comparison [11]. Similar to the case in [7], this network analyzer is quite suitable for measurement related to an antenna without an integrated receiver, but for the measurement related to antenna-receiver integration, suitable adoption of the frequency synthesis approach is required.

The measurement configuration of [21] is another approach aiming to solve the antenna-receiver integrated measurement. The basic idea is to generate a pair of coherent RF and LO sources by using a harmonic-mixer-based phase-locked loop (PLL), followed by a cascading frequency multiplier to terahertz output frequency. A microwave synthesizer is used to provide the pumping of the harmonic mixers in a PLL pair, and the $10-\mathrm{MHz}$ reference signal is multiplied to generate the reference signal for the LO PLL, the RF PLL, second down-conversion pumping, and phasor comparison. The arrangement of different reference signals for frequency synthesis is mainly due to the limited harmonic number suitable for frequency multiplication to terahertz frequency. The main disadvantage of this configuration is the opposite sidebands of the RF and LO signals in the PLL, and the resulting IF leads to additive phase drifting, which will be discussed later. The measurement configurations in [18]-[20] are basically the hybrid types of [7] and [21].

Based on the above discussion, the principle to simplify the frequency synthesis and down-conversion to fulfill the antenna-receiver integration measurement can be summarized as follows.

1) Try to synthesize both RF and LO signal frequencies by the same signal source. For $W$ - or $F$-band signal sources, which need a harmonic mixer in the PLL, a microwave synthesizer to pump the harmonic mixer with frequency $f_{\text {syn }}$ and an 80-110-MHz reference signal with frequency $f_{\text {REF }}$ distributed by power dividers are required.

2) Arrange the RF and LO PLL on the same sideband to avoid phase drifting enlargement.

3) For the receiver under test, the frequency of the IF signal may be too high to compare the amplitude and phase directly, second down conversion is required. To ensure coherence, the second-stage mixing pumping frequency and the second IF frequency should be $f_{\mathrm{syn}}$ and $f_{\mathrm{REF}}$, respectively.

Based on the above principle, a new measurement arrangement is proposed, as shown in Fig. 1. In the configuration, the SIS mixer based heterodyne receiver, including the front-end optics and the LO, is the device-under-test. $W$ - or $F$-band Gunn oscillators are used as the fundamental frequency sources to generate RF signal and LO signal for the receiver. The frequency relationship of this measurement arrangement is described as follows.

Let $f_{\text {osc,RF }}$ and $f_{\text {osc,LO }}$ be the frequencies of the phaselocked oscillators for RF and LO sources, and let $f_{\mathrm{syn}}$ and $f_{\mathrm{REF}}$ be the frequencies of the synthesizers for the PLL harmonicmixing pumping LO source and reference source. One can then express the frequencies as

$$
\begin{aligned}
f_{\mathrm{osc}, \mathrm{RF}} & =n_{p, \mathrm{RF}} f_{\mathrm{syn}}+f_{\mathrm{REF}} \\
f_{\mathrm{osc}, \mathrm{LO}} & =n_{p, \mathrm{LO}} f_{\mathrm{syn}}+f_{\mathrm{REF}} \\
f_{\mathrm{RF}} & =m_{\mathrm{RF}} f_{\mathrm{osc}, \mathrm{RF}} \\
f_{\mathrm{LO}} & =m_{\mathrm{LO}} f_{\mathrm{osc}, \mathrm{LO}} .
\end{aligned}
$$

In (1), $n_{p, \mathrm{RF}}$ and $n_{p, \mathrm{LO}}$ are the harmonic numbers of the harmonic mixers in the RF and LO PLLs, respectively. In (2), $m_{\mathrm{RF}}$ and $m_{\mathrm{LO}}$ are the multiplying factors of the frequency multipliers for the RF and LO sources, respectively. Please note that, in (1), the PLL is arranged at the same sideband, and the upper sideband is expressed in the formulation. This arrangement is to minimize the harmonic difference in the IF output of the receiver under test.

The RF and LO frequency difference should be within the heterodyne receiver IF frequency passband. From (1) and (2), one can get

$$
\begin{aligned}
f_{\mathrm{IF}} & =\left|f_{\mathrm{RF}}-f_{\mathrm{LO}}\right| \\
& =\left|\left(m_{\mathrm{RF}} n_{p, \mathrm{RF}}-m_{\mathrm{LO}} n_{p, \mathrm{LO}}\right) f_{\mathrm{syn}}+\left(m_{\mathrm{RF}}-m_{\mathrm{LO}}\right) f_{\mathrm{REF}}\right| .
\end{aligned}
$$

Since $n_{p, \mathrm{RF}}, n_{p, \mathrm{LO}}, m_{\mathrm{RF}}$, and $m_{\mathrm{LO}}$ are all integers, and the IF signal of the receiver is only down-converted once, the best 


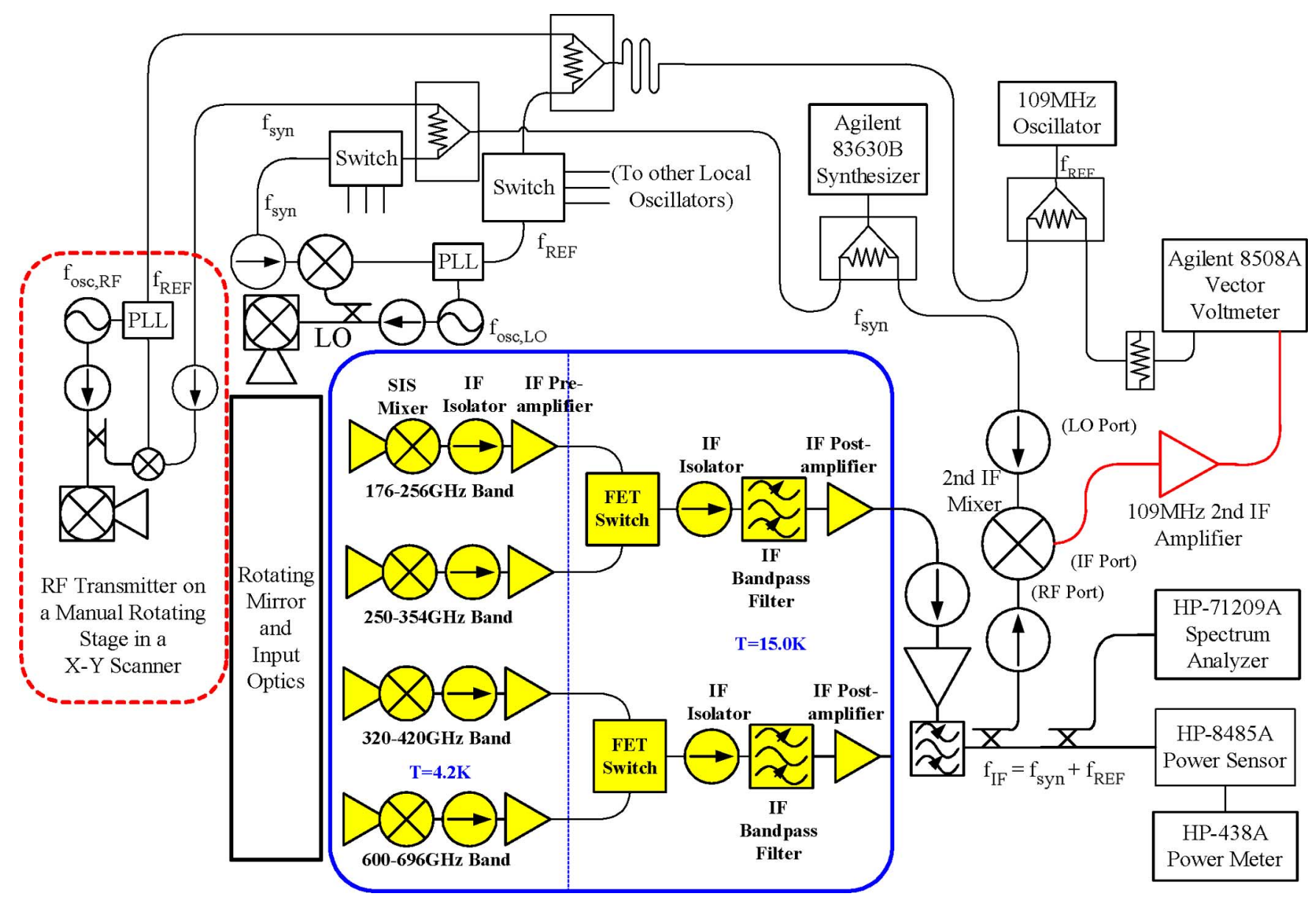

Fig. 1. System block diagram of the submillimeter-wave heterodyne receiver field pattern measurement arrangement.

way is to choose the IF frequency to be equal to $f_{\mathrm{syn}}+f_{\mathrm{REF}}$. The reference frequency based on the above-described principle is much lower than the IF frequency. Therefore,

$$
f_{\mathrm{IF}, L} \leq f_{\mathrm{syn}} \leq f_{\mathrm{IF}, H}
$$

and

$$
\begin{aligned}
\left|m_{\mathrm{RF}} n_{p, \mathrm{RF}}-m_{\mathrm{LO}} n_{p, \mathrm{LO}}\right| & =1 \\
\left|m_{\mathrm{RF}}-m_{\mathrm{LO}}\right| & =1 .
\end{aligned}
$$

$f_{\mathrm{IF}, L}$ and $f_{\mathrm{IF}, H}$ in (4) are the lower and higher edges of the IF passband. Since (5) and (6) represent the harmonic difference of the synthesizer and the phase-locked reference signal sources in the heterodyne receiver output, and these differences are both equal to 1 , one can call this approach the "unitary harmonic difference" method.

In operation, one of the four frequency bands in the receiver system to be measured can be selected by the microwave switches and the rotating optical mirror. An open-ended waveguide antenna with suitable aperture to keep single mode propagation is installed at the output of the RF transmitter when measuring the different frequency bands.

Concerning the mapping approaches, we choose conventional motorized $X Y$ scanning. Considering the $90^{\circ}$ rotation on the polarization of the different frequency bands, an additional manual rotation stage is used for installing the RF transmitter. For the application to other situations, for example, measuring the large-aperture reflector antenna, the proposed frequency synthesis approach can be used along with an innovative hologram-based CATR for large-aperture antenna measurement [13]-[16].

\section{Phase Stability Consideration}

The phase fluctuation of the measurement system can be analyzed as the following. Consider the signal frequencies existing in the beam pattern system as $f_{\mathrm{RF}}, f_{\mathrm{LO}}, f_{\mathrm{IF}}, f_{\mathrm{syn}}$, and $f_{\mathrm{REF}}$ with the corresponded phase errors $\Delta \theta_{\mathrm{RF}}, \Delta \theta_{\mathrm{LO}}$, $\Delta \theta_{\mathrm{IF}}, \Delta \theta_{2 \mathrm{ndIF}}, \Delta \theta_{\mathrm{syn}}$, and $\Delta \theta_{\mathrm{REF}}$. In addition, the phase variations induced by the cable length variation due to ambient temperature change are denoted as $\Delta \theta_{\mathrm{syn}, \mathrm{RF}}, \Delta \theta_{\mathrm{syn}, \mathrm{LO}}$, $\Delta \theta_{\mathrm{syn}, \mathrm{IF}}, \Delta \theta_{\mathrm{REF}, \mathrm{RF}}, \Delta \theta_{\mathrm{REF}, \mathrm{LO}}$, and $\Delta \theta_{\mathrm{REF}, \mathrm{IF}}$. The phase errors introduced by the phase-locked circuits of the RF and LO modules are denoted as $\Delta \theta_{\mathrm{PLL}, \mathrm{RF}}$ and $\Delta \theta_{\mathrm{PLL}, \mathrm{LO}}$.

According to (1), since both the RF and LO sources are phase locked to the upper sideband of the harmonic mixer, the phase fluctuations of RF and LO sources can be expressed as

$$
\begin{aligned}
\Delta \theta_{\mathrm{RF}}=m_{\mathrm{RF}} & n_{p, \mathrm{RF}}\left(\Delta \theta_{\mathrm{syn}, \mathrm{RF}}+\Delta \theta_{\mathrm{syn}}\right) \\
& \left.+\left(\Delta \theta_{\mathrm{REF}, \mathrm{RF}}+\Delta \theta_{\mathrm{REF}}\right)+\Delta \theta_{\mathrm{PLL}, \mathrm{RF}}\right] \\
\Delta \theta_{\mathrm{LO}}=m_{\mathrm{LO}}[ & n_{p, \mathrm{LO}}\left(\Delta \theta_{\mathrm{syn}, \mathrm{LO}}+\Delta \theta_{\mathrm{syn}}\right) \\
& \left.+\left(\Delta \theta_{\mathrm{REF}, \mathrm{LO}}+\Delta \theta_{\mathrm{REF}}\right)+\Delta \theta_{\mathrm{PLL}, \mathrm{LO}}\right] .
\end{aligned}
$$


From (5) and (6), the phase fluctuation of the IF signal is

$$
\begin{aligned}
\Delta \theta_{\mathrm{IF}}=\mid & \left|\Delta \theta_{\mathrm{RF}}-\Delta \theta_{\mathrm{LO}}\right| \\
= & \mid\left(m_{\mathrm{RF}} n_{p, \mathrm{RF}} \Delta \theta_{\mathrm{syn}, \mathrm{RF}}-m_{\mathrm{LO}} n_{p, \mathrm{LO}} \Delta \theta_{\mathrm{syn}, \mathrm{LO}}\right) \\
& +\Delta \theta_{\mathrm{syn}}+\left(\Delta \theta_{\mathrm{PLL}, \mathrm{RF}}{ }^{2}+\Delta \theta_{\mathrm{PLL}, \mathrm{LO}}\right)^{1 / 2} \\
& +\left(\Delta \theta_{\mathrm{REF}, \mathrm{RF}}-\Delta \theta_{\mathrm{REF}, \mathrm{LO}}\right) \mid .
\end{aligned}
$$

Similarly, from (5) and (6), the phase fluctuation for the second IF mixer output signal can be expressed as

$$
\begin{aligned}
\Delta \theta_{2 \mathrm{ndIF}}= & \left|\Delta \theta_{\mathrm{IF}}-\left(\Delta \theta_{\mathrm{syn}, \mathrm{IF}}+\Delta \theta_{\mathrm{syn}}\right)\right| \\
= & \mid\left(m_{\mathrm{RF}} n_{p, \mathrm{RF}} \Delta \theta_{\mathrm{syn}, \mathrm{RF}}-m_{\mathrm{LO}} n_{p, \mathrm{LO}} \Delta \theta_{\mathrm{syn}, \mathrm{LO}}\right) \\
& +\Delta \theta_{\mathrm{syn}, \mathrm{IF}}+\left(\Delta \theta_{\mathrm{PLL}, \mathrm{RF}}{ }^{2}+\Delta \theta_{\mathrm{PLL}, \mathrm{LO}}\right)^{1 / 2} \\
& +\left(\Delta \theta_{\mathrm{REF}, \mathrm{RF}}-\Delta \theta_{\mathrm{REF}, \mathrm{LO}}\right) \mid .
\end{aligned}
$$

In the measurement arrangement, the networks distributed to $\mathrm{RF}$ and LO modules for $f_{\text {syn }}$ share a common 96-in-long coaxial cable connected to a power divider and followed by a pair of cables with the same length of $24 \mathrm{in}$. The link for $f_{\text {syn }}$ to the second IF mixer is directly connected after the first-stage power divider, which means $\Delta \theta_{\text {syn,IF }}$ is ignored. Since all the cables are operated under the same environment with the same ambient temperature variation, one can assume $\Delta \theta_{\mathrm{syn}, \mathrm{RF}}=\Delta \theta_{\mathrm{syn}, \mathrm{LO}}$. In addition, the cable lengths of $\mathrm{RF}$ and $\mathrm{LO}$ reference signals are the same, or $\Delta \theta_{\mathrm{REF}, \mathrm{RF}}=\Delta \theta_{\mathrm{REF}, \mathrm{LO}}$. The second IF phase variation is then

$$
\Delta \theta_{2 \mathrm{ndIF}}=\left|\Delta \theta_{\mathrm{syn}, \mathrm{RF}}+\left(\Delta \theta_{\mathrm{PLL}, \mathrm{RF}}^{2}+\Delta \theta_{\mathrm{PLL}, \mathrm{LO}}^{2}\right)^{1 / 2}\right|
$$

From the phase-noise measurement on the phase-locked Gunn oscillator, the phase noise of the phase-locked circuit is approximately $2.0 \circ$. The phase stability is approximately $3.0^{\circ}$ at $26.5 \mathrm{GHz}$ for a 36 -in-long cable, ${ }^{2}$ which corresponds to $2.15^{\circ}$ at $5.74 \mathrm{GHz}$ for a 120 -in-long cable. Therefore, from (10), the second IF output phase fluctuation is approximately $4.97^{\circ}$.

For the amplitude fluctuation, it is basically due to the bias voltage variation controlled by the PLL and the size of the Gunn oscillator cavity resonator changing with temperature. It is believed the amplitude fluctuation can be minimized by proper temperature control, and it should be small if the Gunn oscillator is turned on for a long time to be thermally balanced to the ambient temperature.

For comparative purposes, the measurement arrangement is analyzed such as that in [21]. The arrangement requires RF and LO sources phase locked at two opposite sidebands, and the reference signals for RF, LO, and IF are 120, 200, and $240 \mathrm{MHz}$, respectively. The microwave synthesizer signal $f_{\text {syn }}$ in the RF and LO signals is cancelled by the submillimeter-wave mixer and the IF frequency $f_{\mathrm{IF}}$ is

$$
f_{\mathrm{IF}^{\prime}}=m_{\mathrm{RF}} f_{\mathrm{REF}^{\prime}, \mathrm{RF}}+m_{\mathrm{LO}} f_{\mathrm{REF}^{\prime}, \mathrm{LO}} .
$$

It is clear that the phase fluctuation at IF in [21] is additive. In addition, the 3-GHz LO signal for the second IF mixer, the reference signals of RF and $\mathrm{LO}$, and the reference signal for the

${ }^{2}$ W. L. Gore PHASEFLEX series microwave cable assembly
TABLE I

HARMONIC NUMBERS AND SIGNAL FREQUENCIES FOR UNITARY HARMONIC DIFFERENCE BEAM PATTERN MEASUREMENT

\begin{tabular}{l|c|c|c|c}
\hline \multicolumn{1}{c|}{ Band } & $176-256 \mathrm{GHz}$ & $250-354 \mathrm{GHz}$ & $320-420 \mathrm{GHz}$ & $600-696 \mathrm{GHz}$ \\
\hline $\mathrm{m}_{\mathrm{LO}}$ & 2 & 3 & 4 & 6 \\
\hline $\mathrm{m}_{\mathrm{RF}}$ & 3 & 4 & 5 & 7 \\
\hline $\mathrm{f}_{\mathrm{Gunn}, \mathrm{LO}}(\mathrm{GHz})$ & $88-128$ & $83-118$ & $82-105$ & $100-120$ \\
\hline $\mathrm{f}_{\mathrm{Gunn}, \mathrm{RF}}(\mathrm{GHz})$ & $58.6-85.3$ & $62.5-88.5$ & $66-86$ & $86-103$ \\
\hline $\mathrm{n}_{\mathrm{LO}}$ & 22 & 21 & 19 & 20 \\
\hline $\mathrm{n}_{\mathrm{RF}}$ & 15 & 16 & 15 & 17 \\
\hline $\mathrm{f}_{\text {syn }}(\mathrm{GHz})$ & $4.0-6.0$ & $4.0-6.0$ & $4.0-6.0$ & $4.0-6.0$ \\
\hline $\mathrm{f}_{\mathrm{REF}}(\mathrm{MHz})$ & 109 & 109 & 109 & 109 \\
\hline $\mathrm{RF}$ Output Antenna & WR-3 & WR2.2 & WR 1.8 & WR 1.2 \\
\hline \multicolumn{5}{|c|}{ Example of Frequency Sets } \\
\hline $\mathrm{f}_{\mathrm{LO}}(\mathrm{GHz})$ & 244.418 & 343.677 & 414.636 & 689.454 \\
\hline $\mathrm{f}_{\mathrm{RF}}(\mathrm{GHz})$ & 250.077 & 349.236 & 409.295 & 683.823 \\
\hline $\mathrm{f}_{\mathrm{Gunn}, \mathrm{LO}(\mathrm{GHz})}$ & 122.209 & 114.559 & 103.659 & 114.909 \\
\hline $\mathrm{f}_{\mathrm{Gunn}, \mathrm{RF}}(\mathrm{GHz})$ & 83.359 & 87.309 & 81.859 & 97.689 \\
\hline $\mathrm{f}_{\text {syn }}(\mathrm{GHz})$ & 5.55 & 5.45 & 5.45 & 5.74 \\
\hline
\end{tabular}

vector voltmeter comparison are through frequency multiplying of a 10-MHz signal. If this $10-\mathrm{MHz}$ signal drifts by temperature even with a very small value, then the second IF output phase fluctuation will be amplified. Accurate environmental temperature control is, therefore, required.

When scanning the field of the beam pattern, there are necessarily moving or flexing cables. The phase variation of these flexing cables may introduce phase error between the LO and $\mathrm{RF}$ source in the measured data. Considering the extreme case of the cable carrying $f_{\text {syn }}$, the phase change is $\pm 1.0^{\circ}$ for one bend around 2.25 -in $(57 \mathrm{~mm})$ radius, which corresponds to $\pm 45.0^{\circ}$ for measurement at $250 \mathrm{GHz}$ or $\pm 120.0^{\circ}$ at $685 \mathrm{GHz}$. However, during the measurement, the flexing cable is bent with a radius much larger than $57 \mathrm{~mm}$, and the scanning distance is not larger than $150 \mathrm{~mm}$, thus the corresponding phase change can be negligible. For the Gaussian beam measurement far from its beam waist, the phase variation at the scanning plane will be quite large, which will also make this cable bend effect much smaller. For measuring the antenna patterns with expected flat phase profile or with large scanning area such as a 1-2-m size, the pilot signal phase correction approach introduced in [24] will be very helpful to enhance the measurement accuracy.

\section{Measurement Results}

In our experiment, the multiple channel receiver system of the SMA telescope is used as the receiver under test. Considering all the criteria given in (4)-(6) and the fact of the fixed harmonic number of the LO sources, the allowable harmonic numbers and its corresponding frequencies are listed in Table I.

\section{A. Phase Stability Measurement}

To verify the phase stability formulation described in (10), the 690-GHz heterodyne receiver is tested with the operating frequency listed in Table I. The amplitude and phase fluctuations over a reasonably long time are measured with the RF transmitter mechanical position fixed. The measurement results, as shown in Fig. 2, indicate the amplitude and phase fluctuation is within $\pm 0.2 \mathrm{~dB}$ and $\pm 4.0^{\circ}$ over a 1-h period, respectively. To be noted is that the testing environment is not air-conditioned. Table II shows the stability performance comparison of this measurement arrangement for other published results. 


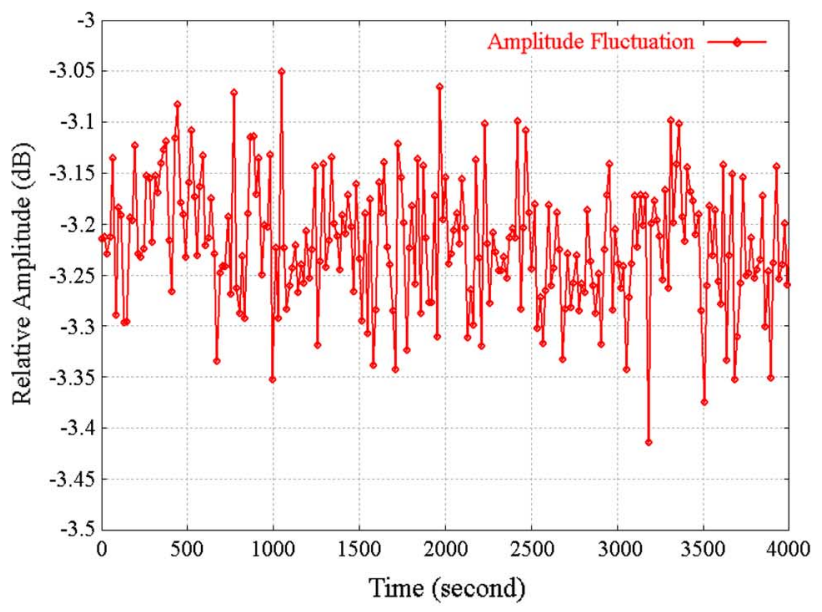

(a)

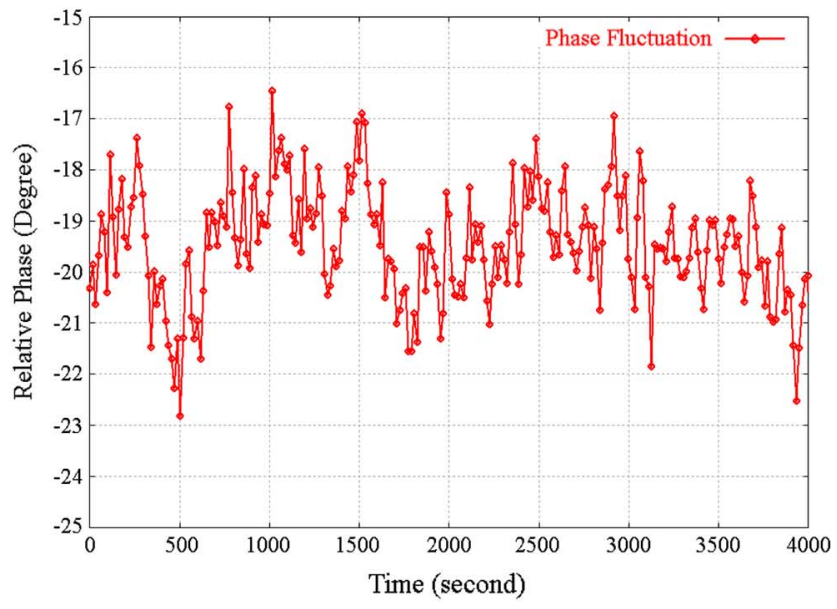

(b)

Fig. 2. Measured results of: (a) amplitude and (b) phase fluctuation of the field pattern measurement system at $690 \mathrm{GHz}$.

TABLE II

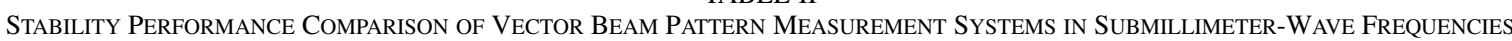

\begin{tabular}{c|c|c|c|c|c|c}
\hline \multirow{2}{*}{ RF Frequency } & \multicolumn{2}{|c|}{ Fluctuation } & \multirow{2}{*}{ SNR } & VNA / Receiver Type & Mapping Type & Ref. \\
\cline { 2 - 4 } & Amplitude & Phase & & & & \\
\hline $345 \mathrm{GHz}$ & - & - & $50-60 \mathrm{~dB}$ & AB Millimetre & X-Y Scanning & {$[9]$} \\
\hline $840 \mathrm{GHz}$ & - & - & $60 \mathrm{~dB}$ & AB Millimetre & X-Y-Z Scanning & {$[10]$} \\
\hline $652 \mathrm{GHz}$ & - & - & $80 \mathrm{~dB}$ & AB Millimetre & X-Y Scanning & {$[11]$} \\
\hline $625 \mathrm{GHz}$ & - & - & $60 \mathrm{~dB}$ & AB Millimetre & X-Y Scanning & {$[13]$} \\
\hline $119 \mathrm{GHz}$ & $0.8 \mathrm{~dB}$ & $10^{\circ}$ & $32 \mathrm{~dB}$ & HP-8510 & Hologram CATR & {$[14]$} \\
\hline $654 \mathrm{GHz}$ & $3 \mathrm{~dB}$ & $40^{\circ}$ & $32-36 \mathrm{~dB}$ & AB Millimetre & Hologram CATR & {$[16]$} \\
\hline $322 \mathrm{GHz}$ & $\pm 0.6 \mathrm{~dB}$ & $\pm 125^{\circ}$ & $52 \mathrm{~dB}$ & AB Millimetre & Hologram CATR & {$[17]$} \\
\hline $200-700 \mathrm{GHz}$ & $0.1 \mathrm{~dB}$ & $4^{\circ}$ & $80 \mathrm{~dB}$ & AB Millimetre & X-Y Scanning & {$[18]$} \\
\hline $211 \mathrm{GHz}$ & - & $\pm 4.0^{\circ}$ & $50 \mathrm{~dB}$ & SIS & X-Y Scanning & {$[19]$} \\
\hline $1028 \mathrm{GHz}$ & $\pm 0.2 \mathrm{~dB}$ & $\pm 15.0^{\circ}$ & $40 \mathrm{~dB}$ & HEB & X-Y-Z Scanning & {$[22]$} \\
\hline $1464 \mathrm{GHz}$ & $\pm 0.15 \mathrm{~dB}$ & $\pm 7.5^{\circ}$ & $25 \mathrm{~dB}$ & HEB & X-Y Scanning & {$[23]$} \\
\hline $683.62 \mathrm{GHz}$ & $\pm 0.2 \mathrm{~dB}$ & $\pm 4.0^{\circ}$ & $43 \mathrm{~dB}$ & SIS & X-Y Scanning & This work \\
\hline
\end{tabular}

\section{B. 2-D Beam Pattern Contour}

After ensuring the stability of the system, the system is further scanned at the frequency listed in Table I. The optics of the lower frequency receiver bands (176-256 and 250-354 GHz) incorporates the wire grid polarizer, LO coupling mesh, and the turning mirrors. The optics of the higher frequency receiver band in 600-696 GHz is equipped with a Martin-Puplett diplexer (MPD) for LO-RF combining. Details of the receiver optics design are described in [25].

The RF source, which is composed of an 84-110-GHz Gunn oscillator, a WR-8 isolator, a crossguide coupler, and a diode frequency multiplier is mounted on a precision $X-Y$ scanner. An open-ended waveguide with proper aperture size and integrated taper to WR-3 is used as the RF transmitting probe antenna. The frequency multiplier is originally equipped with a single-ended Schottky diode and the original multiplying factor is three. However, all the other harmonics can be generated by the diode [26]. As in [27] and [28], the radiation pattern of the probe antenna is regarded to be isotropic in the scanning area.
TABLE III

SCANNING PARAMETERS OF THE BEAM PATTERn MEASUREMENT

\begin{tabular}{c|c|c|c|c}
\hline & $\begin{array}{c}240 \mathrm{GHz} \\
1-\mathrm{D} \mathrm{Scan}\end{array}$ & $\begin{array}{c}690 \mathrm{GHz} \\
1-\mathrm{D} \mathrm{Scan}\end{array}$ & $\begin{array}{c}240 \mathrm{GHz} \\
\text { 2-D Scan. }\end{array}$ & $\begin{array}{c}690 \mathrm{GHz} \\
\text { 2-D Scan. }\end{array}$ \\
\hline Step (mm) & 1.0 & 0.2 & 1.5 & 1.5 \\
\hline Scanning Range (mm) & 140.0 & 80.0 & $140 \times 140$ & $90 \times 90$ \\
\hline Average Factor & 4 & 4 & 4 & 4 \\
\hline Data Points & 140 & 400 & 8931 & 4557 \\
\hline
\end{tabular}

The total optical path from the RF transmitting probe antenna to the receiver horn, including the reflection within the MPD, is approximately $1600 \mathrm{~mm}$ for the $690-\mathrm{GHz}$ band. The scanning step and points for the RF source are listed in Table III.

Fig. 3 shows the amplitude and phase contour plots of the field pattern of the 600-696-GHz SIS receiver. The MPD and cryogenic optical axis of the system are aligned mechanically prior to the installation. It shows that the system has a 43.5-dB dynamic range for the $690-\mathrm{GHz}$ band, which is comparable to $43 \mathrm{~dB}$ given in [23]. The phase contour is matched with the amplitude contour. 


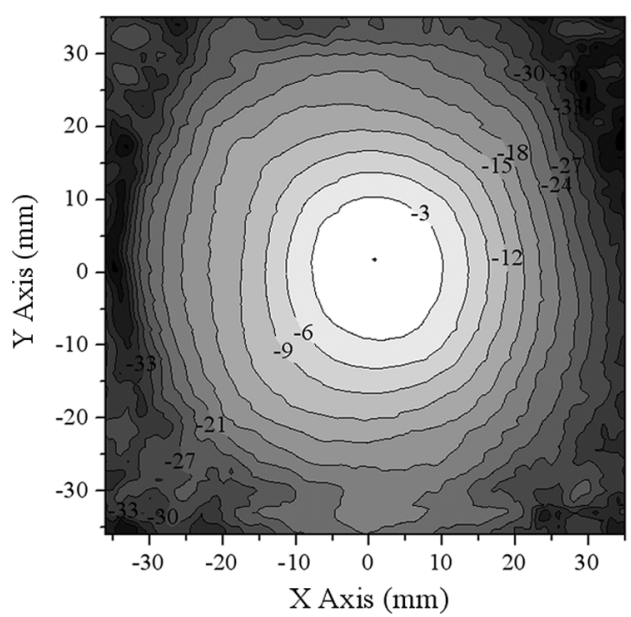

(a)
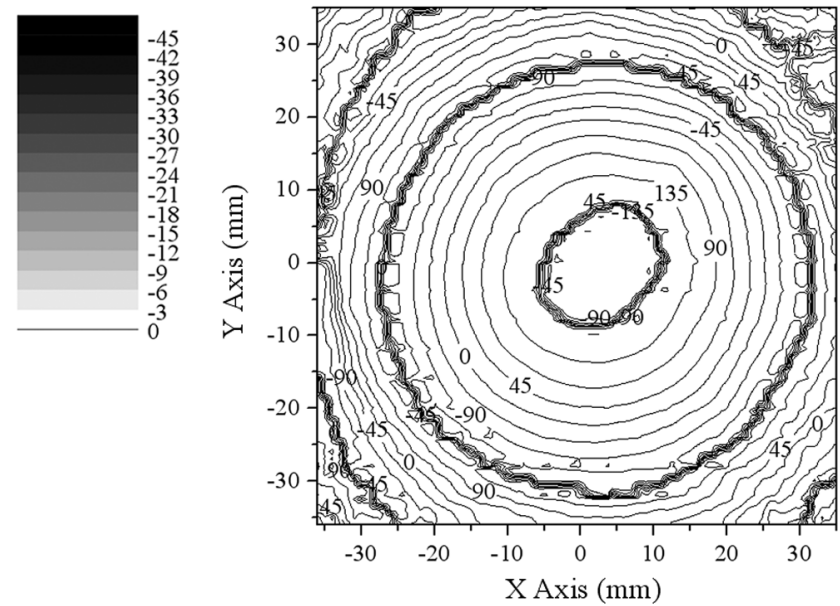

(b)

Fig. 3. Measured results of: (a) amplitude and (b) phase contour of the 600-696-GHz SIS receiver beam pattern.

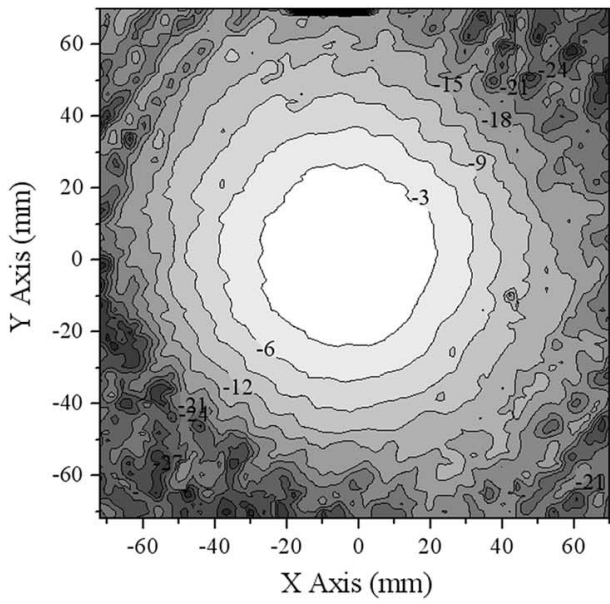

(a)
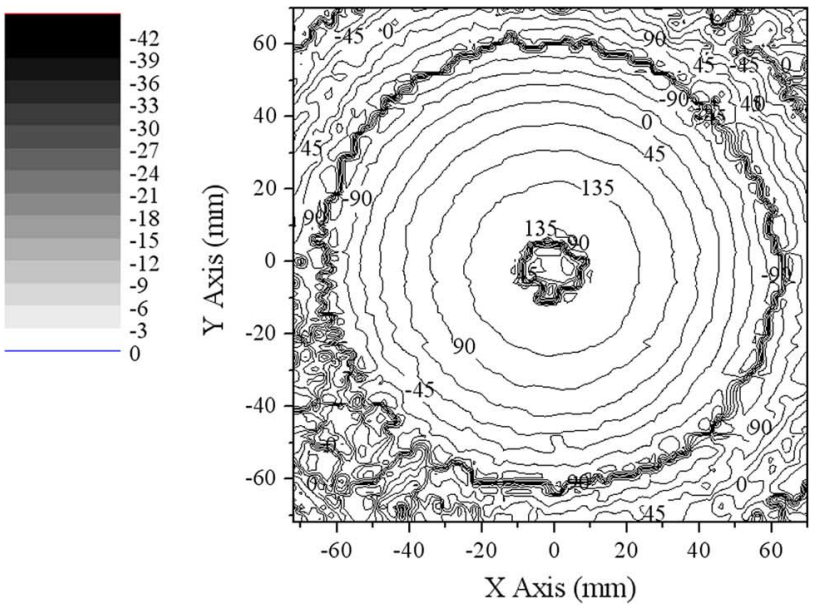

(b)

Fig. 4. Measured results of: (a) amplitude and (b) phase contour of the 176-256-GHz SIS receiver beam pattern.

Fig. 4 shows the amplitude and phase contour plots of the field pattern of the 176-256-GHz SIS receiver. With sufficient $\mathrm{RF}$ power level, the system dynamic range is approximately $47 \mathrm{~dB}$ at $250 \mathrm{GHz}$. It is worth noting that the contour plots at lower frequencies are not as smooth as the measured results of $690 \mathrm{GHz}$, which indicates: 1) stronger background scattering from the metallic mechanical structures of the optics; 2) limited isolation on position-dependent impedance mismatch along the microwave distribution network; and 3) a larger machining error on the receiver corrugated horn antenna and Teflon receiver lens due to larger device size.

\section{Gaussian Optics Parameter Analysis}

In order to ensure that the optics are satisfactory with respect to the design specification, the Gaussian beam parameters are extracted from the $690-\mathrm{GHz}$ measured beam pattern. The detailed formulation for parameter extraction is described as follows.

For a fundamental Gaussian beam without offset and shifting, the amplitude distribution of the vector potential of a paraxial propagated electromagnetic (EM) wave is expressed as [29]

$$
\begin{aligned}
u_{00}(x, y, z)= & \frac{j}{\lambda(z+j b)} \exp \left[-j k \frac{x^{2}+y^{2}}{2(z+j b)}\right] \\
= & \sqrt{\frac{2}{\pi}} \frac{1}{w(z)} \exp \left[-\frac{x^{2}+y^{2}}{w^{2}(z)}\right] \exp (j \phi) \\
& \times \exp \left[-j k \frac{x^{2}+y^{2}}{2 R(z)}\right] \\
= & A_{00}(x, y, z) \exp [j \Psi(x, y, z)]
\end{aligned}
$$

where $w(z)=\sqrt{2 b / k} \sqrt{1+\left(z^{2} / b^{2}\right)}, R(z)=\left(\left(z^{2}+b^{2}\right) / z\right)$, $\phi=\arctan (z / b)$, beam waist radius $w_{o}=\sqrt{2 b / k}, k=2 \pi / \lambda$, and $b$ is the confocal parameter.

At the beam center of $x=y=0, A_{00}(0,0, z)=$ $\sqrt{2 / \pi}(1 / w(z))$, the relative amplitude pattern is given as

$$
A_{R, 00}(x, y, z)=\frac{A_{00}(x, y, z)}{A_{00}(0,0, z)}=\exp \left[-\frac{x^{2}+y^{2}}{w^{2}(z)}\right] .
$$




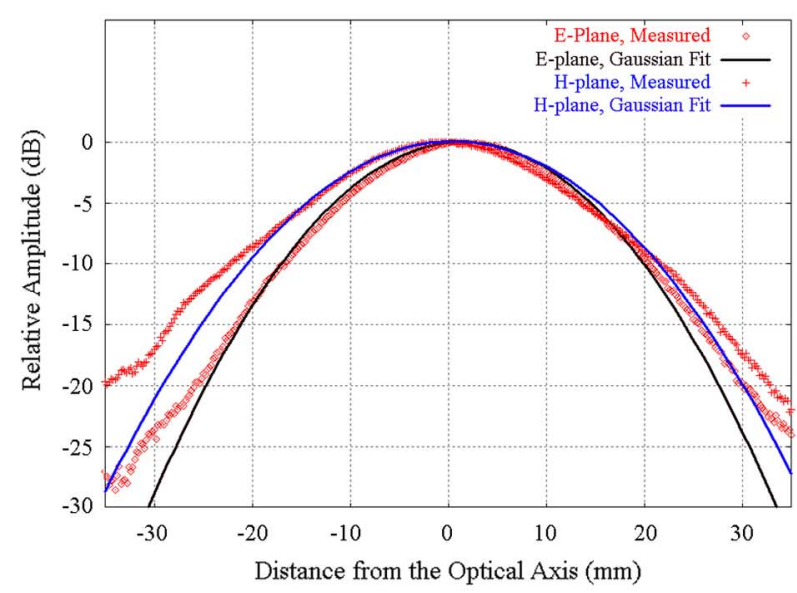

(a)

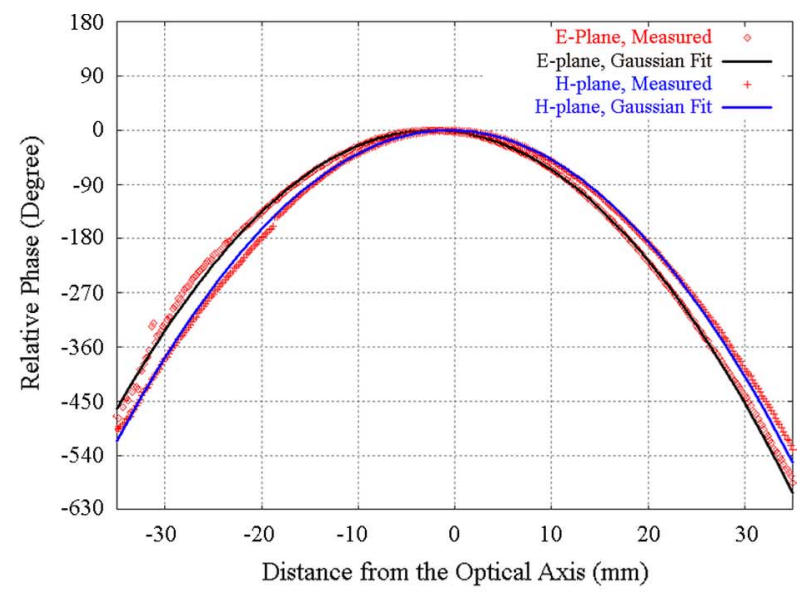

(b)

Fig. 5. Measured results and the Gaussian fit of: (a) amplitude and (b) phase profile of $E$ - and $H$-plane beam pattern.

TABLE IV

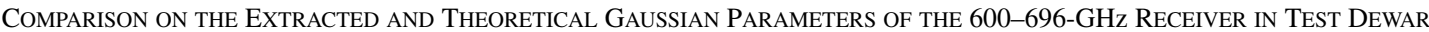

\begin{tabular}{c|c|c|c|c|c|c|c}
\hline & $\begin{array}{c}\text { Amplitude Peak } \\
\left(\mathrm{x}_{\mathcal{c}}, \mathrm{y}_{\mathrm{c}}\right)(\mathrm{mm})\end{array}$ & $\begin{array}{c}\text { Phase Peak } \\
\left(\mathrm{x}_{\mathrm{c}}, \mathrm{y}_{\mathrm{c}}\right)(\mathrm{mm})\end{array}$ & $\mathrm{w}_{\mathrm{o}}(\mathrm{mm})$ & $\mathrm{z}_{\mathrm{w}}(\mathrm{mm})$ & $\delta_{\mathrm{a}}(\mathrm{rad})$ & $\delta_{\mathrm{p}}(\mathrm{rad})$ & $\mathrm{w}(\mathrm{mm})$ \\
\cline { 1 - 5 } $\begin{array}{c}\text { Extracted } \\
\text { E-Plan }\end{array}$ & $(1.60,-1.20)$ & $(-2.40,-0.60)$ & 7.03 & -789 & $1.484 \times 10^{-4}$ & $1.097 \times 10^{-4}$ & 17.22 \\
\cline { 1 - 4 } $\begin{array}{c}\text { Extracted } \\
\text { H-Plan }\end{array}$ & & 6.37 & -840 & $-5.2228 \times 10^{-4}$ & $2.288 \times 10^{-5}$ & 19.48 \\
\hline $\begin{array}{c}\text { Theoretical } \\
\text { Values (I) }\end{array}$ & (N/A) & (N/A) & 7.55 & -783 & $(\mathrm{~N} / \mathrm{A})$ & $(\mathrm{N} / \mathrm{A})$ & 16.31 \\
\hline $\begin{array}{c}\text { Theoretical } \\
\text { Values (II) }\end{array}$ & (N/A) & (N/A) & 6.00 & -890 & (N/A) & (N/A) & 22.34 \\
\hline
\end{tabular}

Note: Theoretical calculation (I) is based on thin lens assumption and the theoretical calculation (II) is based on thick lens assumption.

The beam radius at the scanning plane can then be calculated from the measured relative amplitude pattern as

$$
\begin{aligned}
& w_{x}(z)=\sqrt{\frac{x^{2}}{-\ln \left[A_{R, 00}(x, 0, z)\right]}} \\
& w_{y}(z)=\sqrt{\frac{y^{2}}{-\ln \left[A_{R, 00}(0, y, z)\right]}} .
\end{aligned}
$$

From (12), the relative phase pattern is

$$
\Psi_{R}(x, y, z)=-\frac{k}{2 R(z)}\left(x^{2}+y^{2}\right)
$$

hence, the phase front curvature $R(z)$ can be calculated from the measured relative phase pattern as

$$
R(z)=-\frac{k\left(x^{2}+y^{2}\right)}{2 \Psi_{R}(x, y, z)} .
$$

For practical situations, the beam axis shift and offset should be considered. In other words, the term $x^{2}+y^{2}$ becomes $x^{\prime 2}+y^{\prime 2}+$ $2 R(z) \delta_{x} x^{\prime}+2 R(z) \delta_{y} y^{\prime 2}$, where $x^{\prime}=x-x_{c}, y^{\prime}=y-y_{c}$, and $x_{c}$, $y_{c}$ is the beam center position, and $\delta_{x}$ and $\delta_{y}$ are the beam offsets in $x$ - and $y$-axis. For the receiver system characterization demonstrated here, the optical beam propagation paths along the $E$ - and $H$-plan are different due to the roof mirror pair, which leads to the different truncation of the beam in an orthogonal plan. As shown in Fig. 4, the amplitude pattern is elliptical and the phase pattern is almost circular. The slight misalignment introduced by the multiple reflection within the MPD also leads to a slightly difference positions for the amplitude and phase peaks. Thus, the truncated Gaussian beam through an MPD then becomes

$$
\begin{aligned}
& A_{R, 00}(x, y, z) \\
& \quad=\exp \left[-\left(\frac{x_{a}^{2}}{w_{x}^{2}(z)}+\frac{y_{a}^{2}}{w_{y}^{2}(z)}+k \delta_{x a} x_{a}+k \delta_{y a} y_{a}\right)\right] \\
& \Psi_{R}(x, y, z) \\
& \quad=-k\left(\frac{x_{p}^{2}+y_{p}^{2}}{2 R(z)}+\delta_{x p} x_{p}+\delta_{y p} y_{p}\right) .
\end{aligned}
$$

In (17), $x_{a}=x-x_{c a}, y_{a}=y-y_{c a}, x_{p}=x-x_{c p}, y_{p}=y-y_{c p}$, and $\left(x_{c a}, y_{c a}\right)$ is the position of the amplitude peak and $\left(x_{c p}\right.$, $\left.y_{c p}\right)$ is the position of the phase peak. Therefore, solving $w(z)$ and $R(z)$ from the measured pattern, the shift peak positions $x_{a}$, $y_{a}, x_{p}$, and $y_{p}$ are determined directly from the measured data, and the tilt angles $\delta_{x a}, \delta_{y a}, \delta_{x p}$, and $\delta_{y p}$ are extracted by

$$
\begin{aligned}
& \frac{u_{00}\left(x^{\prime}, 0, z\right)}{u_{00}\left(-x^{\prime}, 0, z\right)}=\exp \left(-2 k \delta_{x a} x_{a}\right) \exp \left(-j 2 k \delta_{x p} x_{p}\right) \\
& \frac{u_{00}\left(0, y^{\prime}, z\right)}{u_{00}\left(0,-y^{\prime}, z\right)}=\exp \left(-2 k \delta_{y a} y_{a}\right) \exp \left(-j 2 k \delta_{y p} y_{p}\right) .
\end{aligned}
$$

The measured Gaussian parameters can then be extracted from (12)-(18). Please note that the $E$ - and $H$-planes are along the $x$ - and $y$-axis, respectively. With the dimensions of the receiver lens-horn combination, one can calculate the theoretical Gaussian beam results of the fundamental mode. Fig. 5 shows the measured beam patterns from [23] of the $E$ - and $H$-planes, with $20-\mu \mathrm{m}$ scanning resolution. The extracted and theoretical Gaussian beam parameters are listed in Table IV. From this 
table, one can see the difference between the theoretical values and measured results of the beam waist radius and positions. It can be regarded as the result of the truncation introduced by the MPD.

\section{CONCLUSION}

In this paper, the optics of a multiple-channel SIS heterodyne receiver has been measured by a new submillimeter-wave phase stable phasor beam pattern measurement configuration. The phase fluctuation at $690 \mathrm{GHz}$ is as small as $\pm 4^{\circ}$ within $1 \mathrm{~h}$. The measurement results indicate this new setup is an efficient tool to characterize and align the optics system of the sub-terahertz SIS receiver. With suitable frequency selection and hardware configuration, this method may be applied to other frequencies as high as $1 \mathrm{THz}$.

\section{ACKNOWLEDGMENT}

The authors would like to thank Dr. R. Blundell, Dr. C.-Y. E. Tong, Dr. S. Paine, and C. Papa, all with the Smithsonian Astrophysical Observatory, Cambridge, MA, for kindly providing the receiver design of the SMA, Dr. M. J. Wang, Academia Sinica Institute of Astronomy and Astrophysics (ASIAA), Taipei, Taiwan, R.O.C., for his contributions of the SIS junctions and mixers, F. Patt, European South Observatory, Garching bei Munich, Germany, for useful discussion, T. S. Wei, ASIAA, Q. Yao, Purple Mountain Observatory, Nanjing, China, S.-H. Chang, ASIAA, S.-W. Chang, ASIAA, and C.-C. Chen, ASIAA, for their technical contributions.

\section{REFERENCES}

[1] P. T. P. Ho, J. M. Moran, and K. Y. Lo, "The Submillimeter Array," Astrophys. J. Lett., vol. 616, no. 1, pt. 2, pp. L1-L6, Nov. 2004.

[2] A. Wooten, Ed., Science With the Acatama Large Millimeter Array. San Francisco, CA: Astronom. Soc. of the Pacific, 2001, pp. 11-24.

[3] J. Inatani, H. Ozeki, R. Satoh, T. Nishibori, N. Ikeda, Y. Fujii, T. Nakajima, Y. Iida, T. Iida, K. Kikuchi, H. Masuko, T. Manabe, S. Ochiai, M. Seta, Y. Irimajiri, Y. Kasai, M. Suzuki, T. Shirai, S. Tsujimaru, K. Shibasaki, and M. Shiotani, "Submillimeter limb-emission sounder JEM/SMILES aboard the Space Station," in Proc. SPIE, Sendai, Japan, Dec. 2000, vol. 4152, Microw. Remote Sens. Atmosphere Environ. II, pp. 243-254.

[4] J. R. Tucker and M. J. Feldman, "Quantum detection at millimetre wavelengths," Rev. Modern Phys., vol. 57, no. 4, pp. 1055-1112, Oct. 1985.

[5] M. J. Wengler and D. P. Woody, "Quantum noise in heterodyne detection," IEEE J. Quantum Electron., vol. QE-23, no. 5, pp. 613-622, May 1987.

[6] B. S. Karasik, M. C. Gaidis, W. R. McGrath, B. Bumble, and H. G. LeDuc, "Low noise in a diffusion-cooled hot-electron mixer at 2.5 THz," Appl. Phys. Lett., vol. 71, no. 11, pp. 1567-1569, Sep. 1997.

[7] A. Feng, D. Dawson, L. Samoska, K. Lee, T. Gaier, P. Kangaslahti, C. Olison, A. Denning, Y. Lau, and G. Boll, "Two-port vector network analyzer measurements in the 218-344- and 356-500 GHz frequency bands," IEEE Trans. Microw. Theory Tech., vol. 54, no. 12, pp. 4507-4512, Dec. 2006.

[8] T. Lüthi, D. Rabanus, U. U. Graf, C. Granet, and A. Murk, "Expandable fully reflective focal-plane optics for millimeter- and submillimeterwave array receivers," Rev. Scientific Instrum., vol. 77, no. 1, Jan. 2006, Paper 014702, 5 pp.

[9] D. Rabanus, C. Granet, A. Murk, and T. Tils, "Measurement of properties of a smooth-walled spline-profile feed horn around $840 \mathrm{GHz}$," Infrared Phys. Technol., vol. 48, no. 3, pp. 181-186, 2006.

[10] A. Murk, P. Fürholz, P. Yagoubov, M. Birk, and G. Wagner, "Near-field antenna measurements for the terahertz limb sounder TELIS," in Proc. Eur. AMTA Symp., 2006, pp. 94-99.

[11] A. Murk, "Characterization of optical components with a $100-800 \mathrm{GHz}$ network analyzer," presented at the IEEE MTT-S Int. Microw. Symp. Workshop, San Francisco, CA, Jun. 2006.
[12] A. Murk, N. Kämpfer, R. Wylde, T. Manabe, M. Seta, and J. Inatani, "Beam pattern measurements of the Submillimeter Limb-Sounder SMILES ambient temperature optics," in 3rd ESA Millimetre Wave Technol. Applicat. Workshop, Espoo, Finland, May 2003, pp. 597-602.

[13] T. Hirvonen, J. Ala-Laurinaho, J. Tuovinen, and A. V. Räisänen, "A compact antenna test range based on a hologram," IEEE Trans. Antenna Propag., vol. 45, no. 8, pp. 1270-1276, Aug. 1997.

[14] J. Mallat, "Vector measurements in MilliLab," Millimetre Wave Lab. Finland, Espoo, Finland, Tech. Note, Aug. 2000.

[15] T. Koskinen, J. Ala-Laurinaho, J. Säily, A. Lönnqvist, J. Häkli, J. Mallat, J. Tuovinen, and A. V. Räisänen, "Experimental study on a hologram-based compact antenna test range at $650 \mathrm{GHz}$," IEEE Trans. Microw. Theory Tech., vol. 53, no. 9, pp. 2999-3006, Sep. 2005.

[16] A. Lönnqvist, T. Koskinen, J. Häki, J. Säily, J. Ala-Laurinaho, J. Mallat, V. Viikari, J. Tuovinen, and A. V. Räisänen, "Hologram-based compact range for submillimeter-wave antenna testing," IEEE Trans. Antenna Propag., vol. 53, no. 10, pp. 3151-3159, Oct. 2005

[17] P. H. Siegel, R. J. Dengler, T. Tsai, P. Goy, and H. Javadi, "Multiple frequency submillimeter-wave heterodyne imaging using an $\mathrm{AB}$ millimetre MVNA," in Proc. IRMMW-THz, Sep. 2005, vol. 2, pp. 576-577.

[18] C.-Y. E. Tong, S. Paine, and R. Blundell, "Near-field characterization of 2-D beam patterns of submillimeter superconducting receivers," in Proc. 5th Int. Space Terahertz. Technol. Symp., Ann Arbor, MI, Apr. 1994, pp. 660-673.

[19] M. T. Chen, C. Y.-E. Tong, L. Chen, S. Paine, and R. Blundell, "Fullwave numerical modeling of near-field beam profiles at 200 and 700 GHz," in Proc. 7th Int. Space Terahertz Tech. Symp., Charlottesville, VA, Mar. 1996, pp. 369-378

[20] M. T. Chen, C. E. Tong, S. Paine, and R. Blundell, "Characterization of corrugated feed horns at 216 and $300 \mathrm{GHz}$," Int. J. Infrared Millim. Waves, vol. 18, no. 9, pp. 1697-1711, Sep. 1997.

[21] C.-Y. E. Tong, D. V. Meledin, D. P. Marrone, S. Paine, H. Gibson, and R. Blundell, "Near-field vector beam measurement at $1 \mathrm{THz}$," IEEE Microw. Wireless Compon. Lett., vol. 13, no. 6, pp. 235-237, Jun. 2003.

[22] C.-Y. E. Tong, D. N. Loudkov, S. N. Paine, D. P. Marrone, and R. Blundell, "Vector measurement of the beam pattern of a $1.5 \mathrm{THz}$ superconducting HEB receiver," in Proc. 16th Int. Space Terahertz Tech. Symp., Göteborg, Sweden, May 2005, pp. 453-456.

[23] Y.-J. Hwang, M.-T. Chen, E. Chung, and T.-H. Chu, "A novel nearfield vector beam pattern measurement system at $690 \mathrm{GHz}$," in Proc. 34th Eur. Microw. Conf., Amsterdam, The Netherlands, Oct. 2004, pp. $557-560$.

[24] J. Säily, P. Eskelinen, and A. V. Räisänen, "Pilot signal based real-time measurement and correction of phase errors caused by microwave cable flexing in planar near-field tests," IEEE Trans. Antenna Propag., vol. 51, no. 2, pp. 195-200, Feb. 2003.

[25] S. Paine, D. C. Papa, R. L. Leombruno, X. Zhang, and R. Blundell, "Beam waveguide and receiver optics for SMA," in Proc. 5th Int. Space Terahertz Technol. Symp., Ann Arbor, MI, Apr. 1994, pp. 811-823.

[26] C.-Y. E. Tong, 1998, private communication.

[27] A. D. Yaghjian, "Approximate formulas for the far field and gain of open-ended rectangular waveguide," IEEE Trans. Antennas Propag., vol. AP-32, no. 4, pp. 378-384, Apr. 1984.

[28] Y. Fujino and C.-Y. E. Tong, "Analysis of an open-end waveguide as a probe for near field antenna measurement by using TLM method," IEICE Trans. Commun., vol. E77-B, no. 8, pp. 1048-1055, Aug. 1994.

[29] H. A. Haus, "Hermite-Gaussian beams and their transformations," in Waves and Fields in Optoelectronics. Englewood Cliffs, NJ: Prentice-Hall, 1984, ch. 5, pp. 108-157.

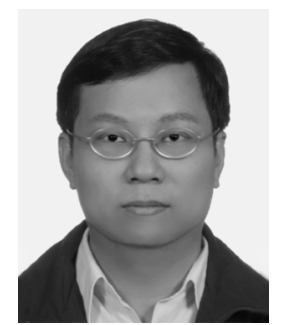

Yuh-Jing Hwang (S'03-M'05) was born in ChangHua, Taiwan, R.O.C., in 1969. He received the B.S., M.S. degree in electrical engineering and Ph.D. degree in communication engineering from National Taiwan University, Taipei, Taiwan, R.O.C., in 1991, 1993 and 2005, respectively.

From 1993 to 1995, he served in the Navy of the R.O.C., as a reserve officer. Since 1995, he has been with the Academia Sinica Institute of Astronomy and Astrophysics (ASIAA), Taipei, Taiwan, R.O.C., as a Microwave Engineer, where he is currently an Assistant Research Fellow. His current research interest is the system integration of low-noise submillimeter-wave SIS heterodyne receivers, antenna measurement techniques in millimeter waves, and millimeter-wave monolithic integrated circuit design. 


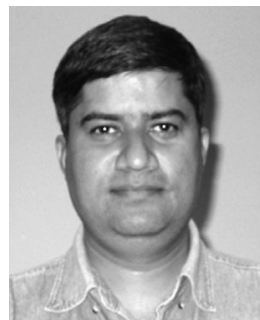

Ramprasad Rao received the B.S.E.E. from the Indian Institute of Technology, Madras, Chennai, India, in 1991, and the Ph.D. degree in astronomy from the University of Illinois at Urbana-Champaign, in 1999.

$\mathrm{He}$ is currently an Engineer and Scientist with the Academia Sinica Institute of Astronomy and Astrophysics, Hilo, HI, where he is involved with the Submillimeter Array. His current research involves the study of magnetic fields in the star formation process in astrophysics and the development of techniques that enable radio telescopes to detect such magnetic fields using polarimetric techniques.

Dr. Rao was the recipient of a 1999 Grainger Fellowship to pursue post-doctoral studies with the Department of Physics, University of Chicago, Chicago, IL. He was also the recipient of the Submillimeter Array Post Doctoral Fellowship presented by the Harvard-Smithsonian Center for Astrophysics.

Rob Christensen, photograph and biography not available at time of publication.

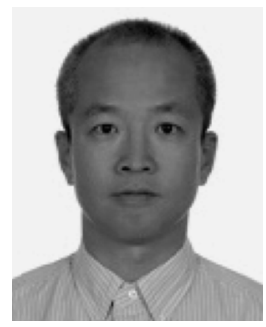

Ming-Tang Chen was born in Tainan, Taiwan, R.O.C. He received the B.S. degree in physics from National Cheng Kung University, Tainan, R.O.C., in 1986, and the M.S. and Ph.D. degrees in physics from the University of Illinois at Urbana-Champaign, in 1990 and 1993, respectively.

From 1993 to 1995, he was Post-Doctoral Fellow with Case Western Reserve University, Cleveland, $\mathrm{OH}$, where he studied pattern formation in helium isotope mixtures in low temperature. In 1995, he shifted his field to join the Institute of Astronomy and Astrophysics, Academia Sinica, Taipei, Taiwan, R.O.C., where he was involved with the development and construction of two antenna elements for the Sub-Millimeter Array. He is currently involved with a cosmology project: the Array for Microwave Background Anisotropy. He has broad interests in various scientific fields in experimental physics ranging from quantum dot to the comic microwave background. His expertise is in the cryogenic technique, quasi-optics, microwave and millimeter-wave instrumentations, and system integration of all kinds.

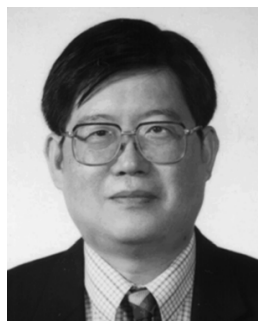

Tah-Hsiung Chu (M'87) received the B.S. degree in electrical engineering from National Taiwan University, Taipei, Taiwan, R.O.C., in 1976, and the M.S. and Ph.D. degrees from the University of Pennsylvania, Philadelphia, in 1980 and 1983, respectively, all in electrical engineering.

From 1983 to 1986, he was a Member of Technical Staff with the Microwave Technology Center, RCA David Sarnoff Research Center, Princeton, NJ. Since 1986, he has been a faculty member with the Department of Electrical Engineering, National Taiwan University, where he is currently a Professor of electrical engineering. His research interests include microwave-imaging systems and techniques, microwave circuit and subsystems, microwave measurements, and calibration techniques. 